\title{
The Design of GPS Information Display System Based on Arduino UNO R3
}

\author{
Rui Liang1, a \\ ${ }^{1}$ Electronic Science teaching and research room, Xi an FanYi University Taiyigong Town, Changan \\ District, Xi'an City, Shanxi Province, China \\ a948793038@qq.com
}

Keywords: Arduino UNO R3; GPS; OLED; positioning system

\begin{abstract}
The global positioning system(GPS)is widely used in the information age of globalization. It has the advantages of stable performance, accurate data and so on. The study of GPS display system has great significance. The Arduino UNO R3 is used as core of control and took advantage of GPS module to position, through the OLED module to display the positioning information. Finally, the GPS positioning information display is realized.
\end{abstract}

\section{Introduction}

This design is based on Arduino UNO R3, including hardware and software in two parts. The hardware part mainly includes two sections. First, the communication of Arduino UNO R3 and GPS module is realized. Second, GPS acquisition information is displayed. The software module is mainly Arduino UNO R3, GPS module serial communication are OLED display.

\section{The Design Principles of the System}

GPS receiver module on the market are similar, one of the biggest difference is the GPS module built-in navigation module and protocol. The design of the GPS module uses the world's leading manufacturers-GPS system professional manufacturing company - Switzerland u-blox company. Module for the U-BLOX-NEO-6M-0-001 is the mobile terminal and car navigation of mainstream module in recent years, also it is small size and cost-effective. The agreement is NMEA-0183 and the United States National Marine Electronics Association for the sea electronic equipment to develop a standard format. Now it has become a unified GPS navigation equipment RTCM standard protocol, the agreement uses ASCLL code to pass GPS positioning information. We call it a frame. The default baud rate of this module is 9600 , the supply voltage is $3.3 \mathrm{v}-5 \mathrm{v}$, which can communicate directly with the Arduino board of 5v power supply. This module can be set parameters through the serial port and can be stored in the EEPROM, while comes with rechargeable backup battery and SMA interface. So, it is highly adaptable.

GPS system consists of three parts: First, the ground control part. This section consists of the master station, which manages the coordination of the entire ground control system. The ground antenna under the control of the master station injects information into the satellite. Also, the monitor station (data automatic collection center) and the communication assistance system belong to its constituent parts. Second, the space part. This section consists of 24 satellites distributed at $60^{\circ}$ intervals on six orbits, of which 24 satellites consisted of 21 working satellites and 3 spare satellites; Third, the user device section. This part due to the data requirements are not the same, such as military and civilian, so the device is different, but the total consists of two modules that named GPS receiver module and satellite antenna module. The relationship between these three parts is shown in Fig 1. 


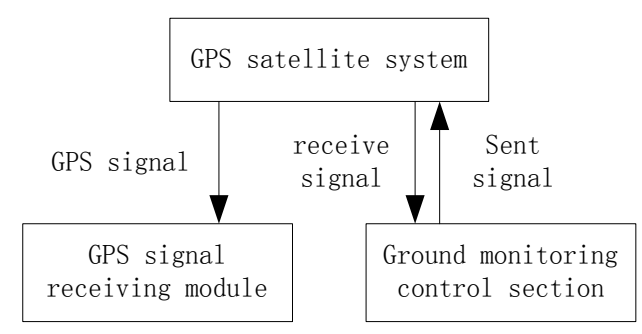

Figure 1. GPS system GPS system composition block diagram

In the distance of $20,000 \mathrm{~km}$ from the ground, 24 GPS satellites to 12 -hour cycle around the Earth to run, so any time and any point can also receive more than 6 GPS satellite positioning information. Only need to have four satellite positioning information GPS receiver module can provide users with time, speed and three-dimensional coordinates and other parameters. Because the spatial position of the satellite is accurate, we can get the distance from the satellite to the receiving module in the observation GPS. Through the three GPS satellites, according to the three-dimensional coordinate distance formula, we can solve the three-dimensional coordinates X, Y, Z. In fact, there is still the fourth unknown, the satellite clock and the user can not always use the clock synchronization, but also need to introduce a clock difference parameter $\triangle \mathrm{t}$, received the fourth satellite data, solution $\triangle \mathrm{t}$. The above calculation can be used to determine the latitude and longitude and height of the receiving module.

The overall design of the block diagram shown in Fig 2, The semaphore of the entire system in the console after the power supply under the command, starting from the satellite signal can be received. Then the midway signal is through the screening process and finally reaching the display. The initial design of the system is achieved by the above processes

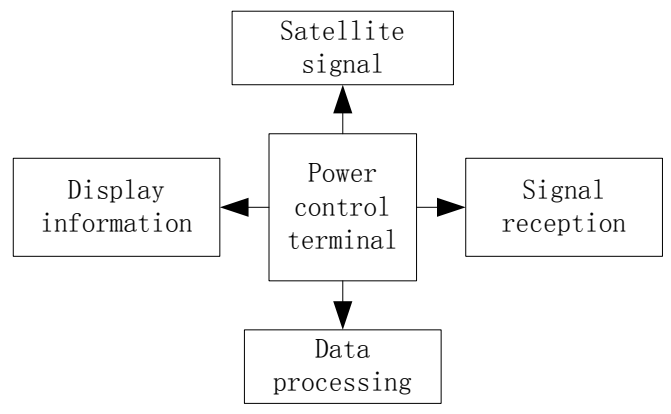

Figure 2. The overall design of the block diagram

\section{The hardware design}

This design is based on Arduino UNO R3 processor, through the serial interface to connect GPS module to receive and process data. The Arduino board interface outputs the data to the OLED liquid crystal display module. The total system hardware consists of the following parts.

Control part (Arduino UNO R3): Arduino UNO R3 board as a microprocessor controls the GPS signal;

Receive part: The GPS receiver module is based on NEO-6M-0-001 as a GPS receiver.

Display part: 1.8OLED display module;

Power supply circuit part: This part specifically includes Arduino dedicated 5v power supply, the wires and welding plates.

The design uses Arduino UNO R3 development board as system micro-processor, controlling GPS data reading and writing conversion. It utilizes the serial port to receive the NEO-6M-0-001 module outputting positioning data, and then extracts the required data that the system first processed. Ultimately, the outcome displays on the OLED. 
In addition to Arduino UNO R3 board, GPS receiver module and OLED display module, the other parts of the system consist of the auxiliary circuit and plug composition. The GPS receiving module based on NEO-6M-0-001 is mainly composed of frequency converter, signal channel, memory, central processor and transmission interface.

Control part (Arduino UNO R3). Arduino is a simple i / o platform based on open source code and the development language and development environment are very simple, easy to understand. It is a tool that can be used to sense and control the real world of physics. It consists of a open source hardware platform of microcontroller-based and a set of development environment for the Arduino board programming. Arduino can be used to develop interactive products, such as it can read a lot of switches and sensor signals, also can control a wide range of electric lights, motors and other physical equipment. The Arduino project can be run either separately or at run time with your computer's program.

The module's gital I / O digital input / output ports are 0-13. Analog I / O Analog input / output ports are 0-5. Support ISP download function. Input voltage: USB without external power supply or external $5 \mathrm{~V} \sim 9 \mathrm{~V}$ DC voltage input. Output voltage: $5 \mathrm{~V}$ DC voltage output and $3.3 \mathrm{~V}$ DC voltage output and external power input. It uses Atmel Atmega328 microprocessor controller. Because of its many supporters, the company has developed 32-bit MCU platform to support Arduino. Several more specific port description: VIN port: VIN is the abbreviation of input voltage, said there is an external power input port. If you do not use USB power supply, the external power supply can be used to provide voltage on this pin, such as battery-powered, which is connected to VIN port and negative connection GND port.

Receive part. The design of the GPS signal receiving module is selected NEO-6M-0-001 as receiving module. This module is produced by the Swiss company u-blox. This module is very small, size $16.0 * 12.2 \mathrm{~mm} * 2.4 \mathrm{~mm}$, hot start and auxiliary start the first positioning time is less than 1 second, $-162 \mathrm{dBm}$ SuperSense capture and tracking sensitivity, with KickStart function, the model can be achieved when the weak start, support AssistNow Online and AssistNow Offline and other A-GPS services, $5 \mathrm{~Hz}$ positioning update rate.

Display part. The design uses OLED (Organic Light Emitting Diode) as a display. OLED has the characteristics of high brightness, low thickness, wide viewing angle, fast response speed, and can be used for flexible panel, wide temperature range, simple structure and simple process. So it is considered the next generation of flat panel display emerging application technology. The screen has the following characteristics:

(1) It has a 1.8-inch OLED screen, and there are yellow blue, white, blue three colors optional, which yellow and blue screen on the 1/4 part of the yellow light, the next 3/4 for the blue light. And the fixed area shows a fixed color, also the color and display area cannot be modified. White is white, which is black and white; Blue is pure blue, which is black and blue. The resolution is $128 * 120$.

(2) The module has a variety of interface modes. OLED bare screen interface includs 6800 and 8080 two parallel interface, 3-wire or 4-wire serial SPI interface, IIC interface (only two lines can control the OLED). These interfaces are through the screen BS0 to BS2.

Overall circuit design. The entire hardware design requires GPS receiver module output signal through a single Arduino board, GPS signal receiving module, 1.8OLED liquid crystal display module, power supply connected to achieve system functions. All modules are connected to the power supply, positive pin: $5 \mathrm{~V}$ or VCC, negative pin: GND, that is, ground pin. The Arduino board controls the GPS module to accept the signal and sends the data to Arduino via pin TX and Arduino pin RXD. Arduino then transfers the data to the OLED display via the other pins connected to the OLED module. The OLED pins and Arduino pins are shown in Table 1. 
Table 1 OLED connection Arduino pin description

\begin{tabular}{|c|c|c|}
\hline VCC & $3.3 / 5 \mathrm{~V}$ & Power supply is 3.3V or 5V \\
\hline SCL & A1 & Clock line \\
\hline SDA & A2 & Data lines \\
\hline RES & A4 & Reset line \\
\hline DC & A3 & Data / command selection line \\
\hline CS & A5 & Chip selection \\
\hline BL & A0 & Backlight switch \\
\hline
\end{tabular}

\section{The Software Design}

Arduino board module through the GPS module to receive the data is transmitted to the integrated controller. After analysis and processing, the processed data is sent to the OLED display. The flow chart is shown in Fig 3.

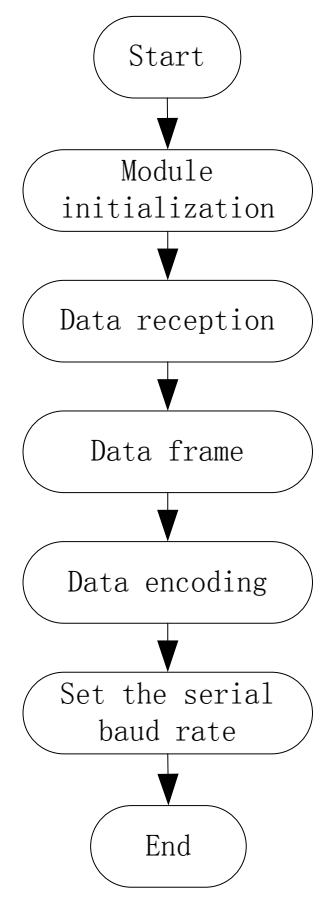

Figure 3. Software flow chart

\section{System Testing and Results Analysis}

The target address for this test is Xi'an FanYi university Room 202, North 8 Apartment. The System serial positioning results are East longitude: 109.361111 and north latitude: 34.028333 . It is converted in radians and angles for East longitude109 $0^{\circ} 13^{\prime \prime}$ and north latitude $34^{\circ} 1^{\prime} 42^{\prime \prime}$. The Internet Baidu website positioning latitude and longitude are respectively 109.015979 and 34.032874 which transformed into radians and angles for East longitude $109^{\circ} 0^{\prime} 57^{\prime \prime}$ and north latitude $34^{\circ} 1^{\prime} 58^{\prime \prime}$. From the results we can see that my design positioning data and Baidu map is almost the same, the error within the allowable range, so the design meets the requirements of the system design.

\section{Conclusion}

The design is based on the Arduino board Arduino UNOR3 processor, through the SiRF Star IIGPS module to receive satellite signals and transmitted to the 1.8OLCD display under the control of Arduino UNOR3. The users can see the field of the latitude and longitude clearly. The system is accurate, easy to operate, practical, strong and has high application value. 


\section{References}

[1] QinH, Cong L, Sun X. Accuracy improvement of GPS/MENS-INS integrated navigation system during GPS signal outage for land vehicle navigation[J]. Journal of Systems Engineering \& Electronics, 2014, 23(2): 256-264.

[2] Koyama Y, Tanaka T. The high-precision measuring method for motorcycle trajectory using GPS[J]. ICCAS-SICE, 2009: 3961-3965.

[3] Klaus Betre. Transmission Characteristics of Marine Differential GPS (DGPS) Stations [EB/OL].http://www.nensch.de/.

[4] Massimo Banzi. Getting Started with Arduino. Edition 2. O’REILLY, Beijing. 2013.

[5] Subhan F, Hasbullah H, Ashraf K. Kalman Filter-Based Hybrid Indoor Position Estimation Technique in Bluetooth Networks[J]. International Journal of Navigation \&Observation, 2013, 2013(1).

[6] Buck Rogers. AG-NAV 2 GPS/DGPS GUIDANCE SYSTEM. Ag Pilot International, 1997, 20(3).

[7] Congwei $\mathrm{Hu}, \mathrm{Wu}$ Chen, Shan Gao. Data Processing For GPS Precise Point Positioning. Transactions of Nanjing University of Aeronautics \& Astronautics, 2015, 22(2).

[8] Qadeer M A, Chandra A, Jain S. Design and implementation of location awareness and sharing system using GPS and 3G/GPRS[J]. International Journal of Multimedia and Ubiquitous Engineering, 2014,7 (4): 125-140.

[9] Dinkar A S, Shaikh S A. Design and Implementation of Vehicle Tracking System Using GPS[J]. Journal of Information Engineering and Applications, 2011,1(3):1-6.

[10] Leiek A. GPS satellite surveying[M]. John Wiley and sons, 2014:13-18. 